\title{
SPECTRAL INVARIANTS OF CONVEX PLANAR REGIONS
}

\author{
SHAHLA MARVIZI \& RICHARD MELROSE
}

\section{Table of contents}

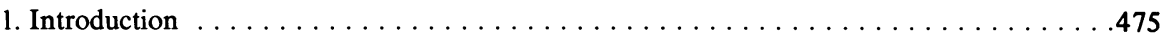

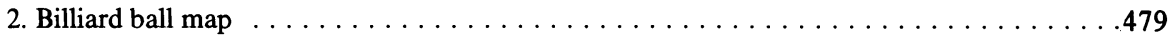

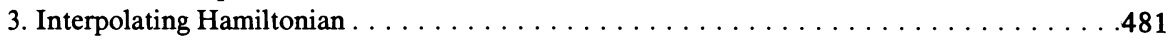

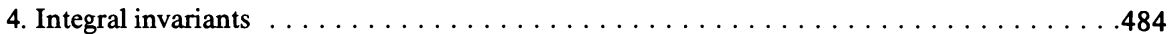

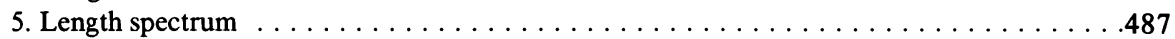

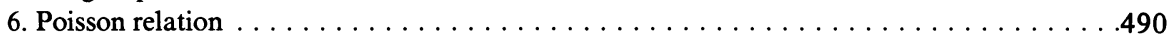

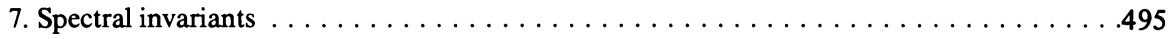

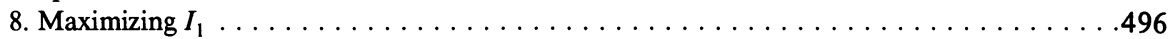

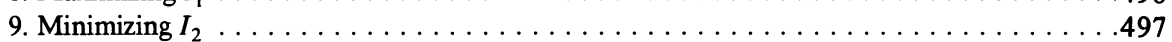

\section{Introduction}

The inverse spectral problem for planar regions was clearly formulated by M. Kac [9]. In this paper, a summary of which appears in [10], we discuss an approach to this problem, and some limited results, for strictly convex planar domains. The objective of inverse spectral theory is the extraction from the spectrum, say of the Dirichlet problem:

$$
\begin{aligned}
& \Delta u=\lambda^{2} u \text { in } \Omega \subset \mathbf{R}^{2}, \\
& \left.u\right|_{\partial \Omega}=0,
\end{aligned}
$$

of some geometric information about the domain $\Omega$ itself. The technique discussed by Kac relies on the fact that the trace of the associated heat equation

$$
\tau(t)=\operatorname{tr}\left(\exp \left(-t \Delta_{D}\right)\right), \quad t>0,
$$

where $\Delta_{D}$ is an unbounded self-adjoint operator on $L^{2}(\Omega)$, is determined by the spectrum (always with multiplicity):

$$
\tau(t)=\sum \exp \left(-\lambda^{2} t\right)
$$

Received May 16, 1982. The first author was supported in part by the NSF under Grant MCS 8108814 , and the second under Grant MCS 8006521. 
As $t \downarrow 0, \tau(t)$ has an asymptotic expansion (see McKean \& Singer [11]),

$$
\tau(t) \sim \sum_{j \geqslant 0} d_{j} t^{-1-1 / 2 j}
$$

where the first few coefficients are known:

$$
d_{0}=(4 \pi)^{-1} \operatorname{Area}(\Omega), \quad d_{1}=\frac{1}{4}(4 \pi)^{-1 / 2} \operatorname{Length}(\partial \Omega), \quad d_{2}=1 / 6, \cdots .
$$

These quantities are examples of spectral invariants. As a simple application (in Kac [9]) the standard isoperimetric inequality (see Osserman [13])

$$
\operatorname{Area}(\Omega) \leqslant \operatorname{Length}(\partial \Omega)^{2} / 4 \pi,
$$

in which there is equality only for the discs, shows that from the spectrum alone it can be determined whether or not the region is a disc.

Now the invariants (1.5) are difficult to determine. We therefore introduce another set of invariants of the problem. These are limited to strictly convex regions and are only shown here to be invariant generically, that is, for an open dense set of regions in a suitable $C^{k}$ topology on the boundary. This set does however contain an open neighborhood of the discs. The new invariants arise in a somewhat subtler way than those in (1.5), and are extracted from

$$
\hat{\sigma}_{D}(t)=\operatorname{tr} \cos \left(\Delta^{1} b^{2} t\right)=\frac{1}{2} \sum \exp (i \lambda t),
$$

where the sum is over all $\lambda$ with $\lambda^{2}$ in the spectrum characterized by (1.1). Thus $\hat{\sigma}_{D}$ is the trace of the solution operator to the wave equation associated to $\Delta_{D}$. For some $\varepsilon(\Omega)>0, \hat{\sigma}_{D}(t)$ is $C^{\infty}$ in $0<t<\varepsilon$ but is singular at $t=0$, having an asymptotic expansion there:

$$
\hat{\sigma}_{D}(t) \sim \sum_{j \geqslant 0} d_{j}^{\prime} t^{-2+j}
$$

The coefficients of this expansion are combinations of the coefficients in (1.4), so give no new information.

As shown in [1], generalizing earlier work of Colin de Verdière, Chazarain [3] and Duistermaat \& Guillemin [5], $\hat{\sigma}_{D}(t)$ is well-defined as a (tempered) distribution on $\mathbf{R}$ and is $C^{\infty}$ away from the length spectrum $\mathcal{L}(\Omega)$ of $\Omega$. That is, away from the set of lengths of closed reflected geodesics of $\Omega$ (including multiples of the length of the boundary). This result was further refined in [6] where the behaviour of the singularity of $\hat{\sigma}_{D}$ near the length of a nondegenerate geodesic (or clean family of geodesics) was analysed. As an application, a simple inverse spectral problem for the ellipse was solved in [7]. Such an assumption of nondegeneracy on the geodesics, whilst generic for convex regions, is very limiting since it precludes even arbitrary small perturbations of 
discs. One of the key results of this paper (Theorem 6.4) shows that for domains satisfying a much weaker noncoincidence condition, $\hat{\sigma}_{D}$ is always singular at a certain subset of $\mathcal{L}(\Omega)$, which we proceed to make precise.

Any closed geodesic on a strictly convex planar region (excluding for the moment the boundary itself from consideration as a geodesic) has associated to it two positive integers, the number $n$ of reflection points and the rotation number $m$ normalized to have $m \leqslant \frac{1}{2} n$. If $\Gamma(m, n)$ denotes the set of such closed geodesics, this set never being empty with these constraints on $m, n$ (see Birkhoff [2]), then

$$
\mathcal{L}(\Omega)=\bigcup_{m, n} L(\Gamma(m, n)) \cup \mathbf{N} L_{\infty},
$$

where $L_{\infty}$ is the length of the boundary, and $L(g)$ for $g \in \Gamma(m, n)$ is the length with multiplicity of the closed geodesic. $\mathcal{L}(\Omega)$ is closed and always has $m L_{\infty}$, for each $m \geqslant 1$, as a limit point from below of $\cup_{n} L(\Gamma(m, n))$. Set

$$
T_{m, n}=\sup L(\Gamma(m, n)), \quad t_{m, n}=\inf L(\Gamma(m, n)),
$$

then as $n \rightarrow \infty$ with $m$ fixed (see Theorem 5.15)

$$
n^{k}\left(T_{m, n}-t_{m, n}\right) \rightarrow 0 \quad \forall k,
$$

and moreover there are constants $c_{k, m}=c_{k, m}(\Omega)$ such that

$$
T_{m, n} \sim m L_{\infty}+\sum_{k=1}^{\infty} c_{k, m} n^{-2 k} \quad(\text { as } n \rightarrow \infty) .
$$

Here $c_{1, m}<0$ always, and these constants or more precisely the $c_{k, 1}$ constitute the new collection of invariants mentioned above.

It is shown below, using in the process a simple lemma on degenerate oscillatory integrals due to Soga [14], that the $c_{k, 1}$ are determined by the spectrum (1.1) for any region which has the property (noncoincidence) that $L_{\infty}$ is not a limit point from below of $\cup_{m^{\prime}>1} L\left(\Gamma\left(m^{\prime}, n^{\prime}\right)\right)$. The more technical component of this proof is the microlocal analysis of the hyperbolic boundary problem:

$$
\begin{aligned}
& \left(D_{t}^{2}-\Delta\right) u(t, x)=0 \quad \text { in } \mathbf{R}_{t} \times \Omega, \\
& u=0 \quad \text { on } \mathbf{R} \times \partial \Omega, \\
& u(0, \cdot)=u_{0}, \quad D_{t} u(0, \cdot)=u_{1} \quad \text { on } \Omega,
\end{aligned}
$$

carried out in [6]; this is used freely.

The constants $c_{k, 1}(\Omega)$ are further reinterpreted in a very useful geometric form. The technical basis for this is the result in symplectic geometry [12] and some remarks in [8]. Thus the behaviour of the geodesics in $\Omega$ is summarized in the billiard ball map, or in the closely related boundary map associated to $\Omega$. 
The latter is a homeomorphism

$$
\delta_{+}: B^{*}(\partial \Omega) \rightarrow B^{*}(\partial \Omega)
$$

of the coball bundle to $\partial \Omega$, the subset of $T^{*} \partial \Omega$ consisting of covectors of length at most one in the induced Riemannian structure on $\partial \Omega$. The map $\delta_{+}$is defined ( $\$ 2$ ) so that if $p_{j} \in T_{\partial \Omega}^{*} \Omega$ are the successive points of a closed geodesic on $\Omega$, then $i^{*}\left(p_{j+1}\right)=\delta_{+} i^{*}\left(p_{j}\right)$, where $i^{*}: T_{\partial \Omega}^{*} \Omega \rightarrow T^{*} \partial \Omega$ is the projection dual to the inclusion of $\partial \Omega$ in $\Omega$. The map $\delta_{+}$is $C^{\infty}$ in the interior of $B^{*} \partial \Omega$ and symplectic there, i.e., preserves the area 2-form. The results of [12] apply to $\delta_{+}$, the proof is outlined below in this special case as Theorem 3.2, to show that there is always a $C^{\infty}$ function $\zeta \in C^{\infty}\left(B^{*} \partial \Omega\right)$ such that

$$
\delta_{+} \equiv \exp \left(-\zeta^{1 / 2} H_{\zeta}\right)
$$

where $H_{\zeta}$ is the Hamilton vector field of $\zeta$, and equality in (1.14) is modulo a $C^{\infty}$ symplectic map which preserves $S_{+}^{*} \partial \Omega$, the counterclockwise component of the boundary of $B^{*} \partial \Omega$, to all orders.

The Taylor series of $\zeta$ at $S_{+}^{*} \partial \Omega$ is uniquely determined by the requirement (1.14). If $d z$ is the 1 -form on the circle $\zeta=t$ (small) normalized by $d z\left(H_{\zeta}\right)=1$, then

$$
I(t)=\int_{\zeta=t} d z
$$

is a $C^{\infty}$ function of $t$ near 0 , with Taylor series at $t=0$ independent of the choice of $\zeta$ satisfying (1.14), i.e.,

$$
I_{k+1}=d^{k} I(0) / d t^{k}=I_{k+1}(\Omega) .
$$

The family of invariants (1.15) is algebraically equivalent to the set of constants $c_{k, 1}$. Explicit formulas (4.6) are obtained for the first two, $I_{1}, I_{2}$, and these are used to find a two parameter family of strictly convex regions which are spectrally determined amongst all regions satisfying the noncoincidence condition. Other spectrally rigid regions can be obtained by considering variational problems involving the area of $\Omega$ in addition to $I_{1}, I_{2}$ and $L_{\infty}$.

In outline this paper proceeds as follows. In the second section formulas for the domain and associated billiard ball map in terms of the curvature function are given. The third section treats the symplectic normal form theorem for the boundary map, which is used in section four to derive formulas for the first two integral invariants, and in section five to relate these invariants to the asymptotic distribution of the lengths of closed geodesics. Section six contains a partial converse to the Poisson relation for convex regions, and this is used in 
section seven to show that the integral invariants are generic spectral invariants. The last two sections, eight and nine, examine the extrema of the first two integral invariants.

The authors wish to thank Victor Guillemin for helpful conversations.

\section{Billiard ball map}

Let $\Omega \subset \mathbf{R}^{2}$ be a smoothly bounded strictly convex region. Thus the boundary $\partial \Omega$ of $\Omega$ is a strictly convex curve, in particular a Jordan curve. We shall give $\partial \Omega$ the counterclockwise orientation. The arc length measure

$$
|d s| \in C^{\infty}\left(\partial \Omega,\left|\Lambda^{1}\right|\right), \quad|d s| \neq 0,
$$

which is fixed in any nonstationary parametrization of $\partial \Omega$

$$
[0 T] \ni t \mapsto(x(t), \quad y(t)) \in \partial \Omega
$$

by $|d s|=\left((\dot{x}(t))^{2}+(\dot{y}(t))^{2}\right)^{1 / 2}|d t|$, can then be extended to a unique 1-form $d s \in C^{\infty}\left(\partial \Omega, \Lambda^{1}\right)$ with $d s>0$ the given orientation. If $p \in \partial \Omega$ is a given point, $\Omega$ can be normalized by a rigid motion so that

$$
p=(0,0), \quad \text { and the positive tangent to } \Omega \text { at } p \text { is }(1,0) \text {. }
$$

The curve $\partial \Omega$, and hence the region $\Omega$, is determined up to a rigid motion by the curvature function. Thus, for $p \in \partial \Omega$ given, let $s=s_{p}$ be the arclength measured from $p$ in the counterclockwise direction. Then $\partial \Omega$ normalized by (2.1) is the curve:

$$
c:[0, L] \ni s \mapsto(x(s), y(s)),
$$

where

$$
x(s)=\int_{0}^{s} \cos (\theta(t)) d t, \quad y(s)=\int_{0}^{s} \sin (\theta(t)) d t
$$

with $\theta(s)$ the angle between tangent and $x$-axis. The curvature function

$$
\kappa(s)=\frac{d}{d s} \theta(s)
$$

is invariantly defined as a function on $\partial \Omega$. Since $\partial \Omega$ is strictly convex, $\kappa>0$. For later purposes it is convenient to introduce angular or curvature coordinates, fixed by the base point $p$,

$$
r=r_{p}=\theta(s)=\int_{0}^{s} \kappa(t) d t
$$

and then to define

$$
\chi(r)=1 / \kappa(s)
$$


(2.7) Proposition. The relationships (2.2)-(2.6) give a 1-1 correspondence between pointed $C^{\infty}$ strictly convex bounded regions normalized by (2.1) and all positive functions $x \in C^{\infty}(\mathbf{R} / 2 \pi \mathbf{Z})$ such that

$$
\begin{gathered}
\text { inf } \chi>0 \\
\int_{0}^{2 \pi} \chi(r) \cos r d r=\int_{0}^{2} \chi(r) \sin r d r=0
\end{gathered}
$$

Proof. If a positive function $\kappa \in C^{\infty}(\mathbf{R} / L \mathbf{R})$ is the curvature function in arclength coordinates of a strictly convex curve of length $L$, then (2.3), (2.5) show that

$$
\int_{0}^{L} \theta(t) d t=2 \pi, \quad \int_{0}^{L} \cos (\theta(t)) d t=\int_{0}^{L} \sin (\theta(t)) d t=0 .
$$

Under the change of variable to $r$ in (2.5) the last two of those conditions reduce to (2.9). The first condition shows that $\chi$ is periodic of period $2 \pi$. Conversely, condition (2.10) shows that (2.3)-(2.6) construct from $\chi \in$ $C^{\infty}(\mathbf{R} / 2 \pi \mathbf{Z})$ a closed strictly convex curve as in (2.1), proving the proposition.

Note that the length of the curve is

$$
L_{\infty}=\int_{0}^{2 \pi} \chi(r) d r
$$

The billiard ball map of the region $\Omega$ describes the relationship of points on the boundary $\partial \Omega$ lying on the same straight line. Let $G$ be the circle bundle over $\partial \Omega$ with fibres the circles of unit tangent vectors to $\mathbf{R}^{2}$ at each point of $\partial \Omega$. Then

$$
G=\partial \Omega \times S^{1}, \quad S^{1}=(\mathbf{R} / 2 \pi \mathbf{Z}) .
$$

The billiard ball map can be viewed as an involution $\beta$ : $G \rightarrow G$, defined by

$$
\beta(x, \theta)=\left(x^{\prime}, \theta^{\prime}\right),
$$

if $\theta^{\prime}=\tau \theta$, using the translation of tangent vectors in $\mathbf{R}^{2}$, and $x, x^{\prime}$ lie on the straight line with direction $\theta$ :

$$
x-x^{\prime}=D \theta, \quad D \in \mathbf{R} .
$$

The circle bundle $G$ projects orthogonally, along the normal bundle to $\partial \Omega$, onto $B^{*} \partial \Omega \subset T^{*} \partial \Omega$, the unit coball bundle to $\partial \Omega$. In fact this projection has two continuous inverses

$$
\mu_{ \pm}: B * \partial \Omega \rightarrow G, \quad \pi \cdot \mu_{ \pm}=\mathrm{Id},
$$

where $\pi$ is the projection and $\mu_{ \pm}$have respectively the inward and outward pointing halves of $G$ as ranges. The composite maps

$$
\delta_{ \pm}: B^{*} \partial \Omega \rightarrow B^{*} \partial \Omega, \quad \delta_{ \pm}=\pi \cdot \beta \cdot \mu_{ \pm}
$$


are symplectomorphisms called the boundary maps-specifically the inward and outward boundary maps - they are inverses of each other.

Next we proceed to compute a formula for $\delta_{ \pm}$in terms of the curvature. If the straight line (2.12) passes through $c(s)=(x(s), y(s))$ and makes an angle $\varphi$, measured inward from the forward tangent at $c(s)$, then $(s, \varphi)$ give global coordinates in $G$, and in terms of the canonical coordinates $(s, \sigma)$ in $T^{*} \partial \Omega$ dual to the arclength,

$$
\pi(x, \varphi)=(s, \sigma) \Leftrightarrow \sigma=\cos \varphi
$$

Moreover,

$$
\tan (\varphi+r)=\frac{y\left(s^{\prime}\right)-y(s)}{x\left(s^{\prime}\right)-x(s)}=\frac{\int_{r}^{r^{\prime}} \chi(t) \sin t d t}{\int_{r}^{r^{\prime}} \chi(t) \cos t d t},
$$

where $r, r^{\prime}$ are the values of the curvature variable at $x=c(s), x^{\prime}=c\left(s^{\prime}\right)$ on the line with angle $\varphi$ at $c(s)$. If $\beta(x, \varphi)=\left(x^{\prime}, \varphi^{\prime}\right)$, then the equality in (2.15) fixes $r^{\prime}$ in terms of $(s, \varphi)$, and clearly

$$
\varphi+r=\varphi^{\prime}+r^{\prime}
$$

To reduce this to a formula for $\delta_{+}$notice that one should take $\varphi \in[0, \pi]$. In particular, if $r$ is the curvature coordinate normalized at $p$, then

$$
\delta_{+}(0, \sigma)=\left(r^{\prime}, \sigma^{\prime}\right)
$$

if

$$
\frac{\left(1-\sigma^{2}\right)^{1 / 2}}{\sigma}=\frac{\int_{r}^{r^{\prime}} \chi(t) \sin t d t}{\int_{r}^{r^{\prime}} \chi(t) \cos t d t}
$$

because of (2.14).

\section{Interpolating Hamiltonian}

As noted above (see for example [6]) the boundary maps $\delta_{ \pm}$are symplectic with respect to the standard symplectic (area) form on $T^{*} \partial \Omega$, that is, $\delta_{ \pm}$ preserve the 2-form

$$
\sin (\varphi) d s \wedge d \varphi=d \sigma \wedge d s .
$$

Note that $\delta_{ \pm}$are not $C^{\infty}$ up to the boundary $S^{*} \partial \Omega$ of $B^{*} \partial \Omega$ but are continuous and fix $S^{*} \partial \Omega$ pointwise. The singularity of $\delta_{ \pm}$at $S^{*} \partial \Omega$ is of square-root type as is clear from (2.16), which shows that $r^{\prime}=R^{\prime}\left(r,(1-\sigma)^{1 / 2}\right)$, with $R^{\prime}$ a $C^{\infty}$ function, $R^{\prime}(r, 0)=0, d R^{\prime}(r, t) / d t>0$ at $t=0$. The precise form of this singularity is described in the theorem below. This is essentially a corollary of the main result of [12], and holds for convex regions in any dimension. For 
completeness a proof is given here for the special case of planar domains. For $\zeta \in C^{\infty}\left(T^{*} \partial \Omega\right)$ we denote by $H_{\zeta}$ the Hamilton vector field of $\zeta$ on $T^{*} \partial \Omega$.

(3.2) Theorem. If $\delta_{ \pm}$are the boundary maps of a strictly convex $C^{\infty}$ planar domain, there exists a $C^{\infty}$ function $\zeta \in C^{\infty}\left(T^{*} \partial \Omega\right)$ which is a defining function for the positive half $S_{+}^{*} \partial \Omega$ of the cosphere bundle such that $\zeta \geqslant 0$ in $B^{*} \partial \Omega$ and

$$
\delta_{ \pm} \cdot \exp \left( \pm \zeta^{1 / 2} H_{\zeta}\right)=\rho_{ \pm}
$$

are $C^{\infty}$ maps near $S_{+}^{*} \partial \Omega \subset B^{*} \partial \Omega$ fixing $S_{+}^{*} \partial \Omega$ to infinite order. The Taylor series at $S_{+}^{*} \partial \Omega$ of $\zeta$ is determined by the requirement (3.3).

As shown in [12], locally near any point of $S_{+}^{*} \partial \Omega, \zeta$ can be chosen so that (3.3) holds with $\rho_{ \pm}=$Id near the point. That is, the boundary maps $\delta_{ \pm}$(and hence the billiard ball map $\beta$ ) are always locally completely integrable. In general it is not possible to choose $\zeta$ so that (3.3) holds in a neighborhood of $S_{+}^{*} \partial \Omega$ with $\rho_{ \pm}=$Id.

Proof of Theorem (3.2). Note that $\pi$ in (2.14) is an isomorphism onto $S^{*} \partial \Omega$, we denote its domain $\pi^{-1}\left(S^{*} \partial \Omega\right)$ by $S^{*} \partial \Omega \subset G$. Again from (2.14) it is clear that if $f \in C^{\infty}(G)$, then $f=\pi^{*} g$, near $S_{+}^{*} \partial \Omega$ with $g \in C^{\infty}\left(T^{*} \partial \Omega\right)$ if and only if $f$ is locally invariant under the involution

$$
\alpha: G \rightarrow G, \quad \alpha(x, \varphi)=(x,-\varphi) .
$$

From (3.3) it would follow that the Taylor series of $\zeta$ at $S^{*} \partial \Omega$ was invariant under $\delta_{ \pm}$. Thus we look first for a Taylor series even under $\alpha$,

$$
g=\sum_{j} g_{j}(s) \varphi^{2 j}
$$

such that $\beta^{*} g$ is also even. If $\beta^{*}(\varphi)=\varphi^{\prime}, \beta^{*}(s)=s^{\prime}$, then

$$
\beta^{*} g=\sum_{j} g_{j}\left(s^{\prime}\right)\left(\varphi^{\prime}\right)^{2 j}=\sum_{k=2}^{\infty} p_{k}(s) \varphi^{k}
$$

From (2.5) it follows that

(3.6) $\varphi^{\prime}=-h(s) \varphi+k(s) \varphi^{2}+O\left(\varphi^{3}\right), \quad s^{\prime}=s+w(s) \varphi+O\left(\varphi^{2}\right)$

with $h(0)>0, w(0)>0$. Consider the term $p_{3}$ in (3.5):

$$
p_{3}(s)=w(s) d g_{1} / d s+2 k(s) g_{1} .
$$

Thus (3.7) shows that the equation $p_{3}(s)=0$ has a unique local solution $g_{1}(s)$ with $g_{1}(0)=1$. Similarly, adding the terms $g_{j}$ to $g$ changes the coefficient $p_{2 j+1}$ in $\beta^{*} g$ by

$$
w(s) \frac{d g_{j}(s)}{d s}+2 j k(s) g_{j}(s)
$$


and leaves the coefficients $p_{2 r}, r<j$, unchanged. Thus, for $j>1, g_{j}$ is uniquely fixed by requiring that $g_{j}(0)=0$ and that $\beta^{*} g$ have no term $\varphi^{2 j+1}$. By induction we can therefore choose $g$ in (3.4) with $g_{1} \neq 0$ and $\beta^{*} g$ even in $\varphi$. Then

$$
t=\frac{1}{2}\left(g+\beta^{*} g\right)
$$

is a Taylor series at $S_{+}^{*} \partial \Omega$ even under both $\beta$ and $\alpha$, since these are both involutions. Choosing $t \in C^{\infty}\left(T^{*} \partial \Omega\right)$ consistent with this Taylor series then satisfies

$$
\delta_{ \pm}^{*} t-t \in C^{\infty}\left(B^{*} \partial \Omega\right) \quad \text { near } S_{+}^{*} \partial \Omega
$$

vanishes to all orders at $S_{+}^{*} \partial \Omega$.

Next solve the equation

$$
H_{t} \tau=1,\left.\quad \tau\right|_{s=0}=0
$$

clearly possible locally since $H_{t}$ is tangent to $S_{+}^{*} \partial \Omega$ and nonzero. This gives local coordinates $(\tau, t)$ in which the symplectic 2 -form $\omega$ on $T^{*} \partial \Omega$ is again $\omega=d t \wedge d \tau$. From (3.9), $H_{t}\left(\delta_{+}^{*} \tau\right)=1+e, e \in C^{\infty}$ vanishing to all orders at $S_{+}^{*} \partial \Omega$. Thus, for some $g^{\prime} \in C^{\infty}(\mathbf{R})$,

$$
\delta_{+}^{*} \tau \equiv \tau+g^{\prime}(t) t^{1 / 2},
$$

modulo a $C^{\infty}$ function vanishing to all orders at $S_{+}^{*} \partial \Omega$. From $(3.6), g^{\prime}(0) \neq 0$, so new symplectic coordinates

$$
\zeta=R(t), \quad z=\tau / R^{\prime}(t)
$$

can be introduced satisfying $R(t) d R / d t=-g^{\prime}(t) t$, i.e.,

$$
R(t)=\left(-2 \int_{0}^{t} g^{\prime}(s) s d s\right)^{1 / 2} .
$$

Thus we have

$$
\delta_{+}^{*} z \equiv z-\zeta^{1 / 2}, \quad \delta_{+}^{*} \zeta \equiv \zeta, \quad \omega=d \zeta \wedge d z
$$

in the sense of Taylor series at $\zeta=0$, i.e., $S_{+}^{*} \partial \Omega$. Since $H_{\zeta}=\partial_{z}$, this leads to (3.3) at least locally near a given point of $S^{*} \partial \Omega$.

The uniqueness of the Taylor series at $S_{+}^{*} \partial \Omega$ follows directly, using the fact that (3.11) must hold in the new coordinates $z^{\prime}, \zeta^{\prime}$. Always in the sense of Taylor series at $\zeta=0$ it follows from $\delta_{+}^{*} \zeta^{\prime} \equiv \zeta^{\prime}$ that $\zeta^{\prime} \equiv w(\zeta)$. Since $H_{\zeta^{\prime}} z^{\prime}=1$, $H_{\zeta} z^{\prime} \equiv w^{\prime}(\zeta)$, so $z^{\prime} \equiv w^{\prime}(\zeta) z$. Thus $\delta_{+}^{*} z^{\prime} \equiv z^{\prime}-w^{\prime}(\zeta) \zeta^{1 / 2}$, i.e., $\zeta^{\prime} \equiv \zeta$. Having constructed $\zeta$ everywhere locally at $S_{+}^{*} \partial \Omega$, this local uniqueness shows that the Taylor series of $\zeta$ satisfying (3.3) exists and is unique around $S_{+}^{*} \partial \Omega$, completing the proof of the theorem. 


\section{Integral invariants}

In this section we examine some properties of the integral invariants (1.15), for a given strictly convex region $\Omega \subset \mathbf{R}^{2}$, and compute explicit formulas (4.8) for the first two of them.

(4.1) Lemma. If $\zeta \in C^{\infty}\left(B^{*} \partial \Omega\right)$ is an interpolating Hamiltonian at $S_{+}^{*} \partial \Omega$ for the boundary map of a strictly convex planar region, then for some $\varepsilon>0$ the circles $A(t)=\{\zeta=t\}, 0<t<\varepsilon$, carry $C^{\infty} 1$-forms $d z, d z\left(H_{\zeta}\right)=1$, and

$$
I(t)=\int_{A(t)} d z \in C^{\infty}([0, \varepsilon)) .
$$

Proof. Since $\zeta$ is a defining function for $S_{+}^{*} \partial \Omega$, the $A(t)$ clearly are circles depending smoothly on $t$ for $t \in[0, \varepsilon), \varepsilon$ small. Again, $H_{\zeta}$ is tangent to each $A(t)$, and $H_{\zeta} \neq 0$, so $d z$ is a $C^{\infty} 1$-form depending smoothly on $t$. Thus (4.2) holds, proving the lemma.

Changing from $\zeta$ to another interpolating Hamiltonian $\zeta^{\prime}, \zeta^{\prime}-\zeta$ always vanishes to all orders at $S_{+}^{*} \partial \Omega$, so the new 1 -form $d z^{\prime}$ differs by $d z^{\prime}-d z$, from the old, and this vanishes to all orders at $S_{+}^{*} \partial \Omega$, as a 1 -form on $B^{*} \partial \Omega$. Thus the new function $I^{\prime}(t)$ is such that $I^{\prime}(t)-I(t)$ vanishes to all orders at $t=0$. In consequence the coefficients in the Taylor series

$$
I_{k+1}=d^{k} I(0) / d t^{k}
$$

are independent of the choice of $\zeta$.

If we take

$$
\zeta=(1-\sigma) \zeta_{1}(s)+(1-\sigma)^{2} \zeta_{2}(s)+O(1-\sigma)^{3},
$$

then in terms of the variable $s$ on $A(t)$, with $\sigma=\sigma(s), \zeta(s, \sigma(s))=t$,

$$
\begin{aligned}
& I_{1}=\int_{0}^{L}\left(\frac{\partial \zeta}{\partial \sigma}\right)^{-1} d s=-\int_{0}^{1} \zeta_{1}^{-1} d s, \\
& I_{2}=\left.\frac{d}{d t} \int_{0}^{L}\left(\frac{\partial \zeta}{\partial \sigma}\right)^{-1} d s\right|_{t=0}=\int_{0}^{L} \zeta_{2} \zeta_{1}^{-3} d s .
\end{aligned}
$$

A $C^{\infty}$ function of $(s, \sigma)$, such as $\zeta$, must be $C^{\infty}$ and even in $\varphi$ across $\varphi=0$, $\sigma=\cos (\varphi)$, i.e.,

$$
\zeta=a_{1}(s) \varphi^{2}+a_{2}(s) \varphi^{4}+O\left(\varphi^{6}\right) .
$$

Therefore $\zeta_{1}=2 a_{1}, \zeta_{2}=\frac{1}{3} a_{1}+4 a_{2}$. We proceed to compute $a_{1}, a_{2}$.

Let $\delta_{+}(s, \sigma)=\left(s^{\prime}, \sigma^{\prime}\right)$. Since (3.3) holds, the Taylor series of $s^{\prime}$ at $\zeta=0$ is given by

$$
s^{\prime}=s-\zeta^{1 / 2} H_{\zeta} s+\frac{1}{2} \zeta H_{\zeta}^{2} s-\frac{1}{6} \zeta^{3 / 2} H_{\zeta}^{3} s+\cdots
$$


Now

$$
\begin{aligned}
& H_{\zeta} s=-\frac{I}{\sin (\varphi)} \frac{\partial \zeta}{\partial \varphi}=-2 a_{1}-\left(4 a_{2}+\frac{1}{3} a_{1}\right) \varphi^{2}+O\left(\varphi^{4}\right), \\
& H_{\zeta}^{2} s=\frac{1}{\sin (\varphi)}\left(\frac{\partial \zeta}{\partial \varphi} \partial_{s}-\frac{\partial \zeta}{\partial s} \partial_{\varphi}\right)\left(\frac{1}{\sin (\varphi)} \frac{\partial \zeta}{\partial \varphi}\right)=4 a_{1} \dot{a}_{1}+O\left(\varphi^{2}\right), \\
& H_{\zeta}^{3} s=\cdots=-8\left(a_{1}^{2} \ddot{a}_{1}+a_{1} \dot{a}_{1}^{2}\right)+O\left(\varphi^{2}\right),
\end{aligned}
$$

where $\dot{a}_{1}$ and $\ddot{a}_{1}$ are respectively the first and second derivatives of $a_{1}$ with respect to $s$. Also

$$
\zeta^{1 / 2}=a_{1}^{1 / 2} \varphi+\frac{1}{2} a_{1}^{-1 / 2} a_{2} \varphi^{3}+O\left(\varphi^{4}\right) .
$$

Inserting these expansions into (4.3) gives

$$
\begin{aligned}
s^{\prime}= & 2 a_{1}^{3 / 2} \varphi+2 a_{1}^{2} \dot{a}_{1} \varphi^{2} \\
& +\left(\frac{1}{3} a_{1}^{3 / 2}+5 a_{1}^{1 / 2} a_{2}+\frac{4}{3} a_{1}^{5 / 2} \dot{a}_{1}+\frac{4}{3} a_{1}^{7 / 2} \ddot{a}_{1}\right) \varphi^{3} \\
& +O\left(\varphi^{4}\right) .
\end{aligned}
$$

Next we compute the same thing geometrically and equate the coefficients of $\varphi^{j}, 1 \leqslant j \leqslant 3$. This gives $a_{1}, a_{2}$ in terms of $\kappa$ and its derivatives. With $\theta(s)=\int_{0}^{s} \kappa(t) d t$, set

$$
\theta(s)=s \theta_{1}+\frac{1}{2} s^{2} \theta_{2}+\frac{1}{6} s^{3} \theta_{3}+\cdots .
$$

Thus

$$
\begin{aligned}
& x(s)=\int_{0}^{s} \cos (\theta(t)) d t=s-\frac{1}{6} s^{3} \theta_{1}^{2}-\frac{1}{8} s^{4} \theta_{1} \theta_{2}+O\left(s^{5}\right), \\
& y(s)=\int_{0}^{s} \sin (\theta(t)) d t=\frac{1}{2} s^{2} \theta_{1}+\frac{1}{6} s^{3} \theta_{2}+\frac{1}{24} s^{4}\left(\theta_{3}-\theta_{1}^{2}\right)+O\left(s^{5}\right) .
\end{aligned}
$$

Then according to (2.15) we have

$$
\tan (\varphi)=\frac{1}{2} s^{\prime} \theta_{1}+\frac{1}{6}\left(s^{\prime}\right)^{2} \theta_{2}+\frac{1}{24}\left(s^{\prime}\right)^{3}\left(\theta_{3}+\theta_{1}^{3}\right)+O\left(s^{\prime}\right)^{4},
$$

and substituting for $s^{\prime}$ from (4.4) and expanding $\tan (\varphi)$ give, after the identification $\theta_{1}=\kappa, \theta_{2}=\dot{\kappa}, \theta_{3}=\ddot{\kappa}$,

$$
a_{1}=\kappa^{-2 / 3}, a_{2}=-\frac{1}{15} \kappa^{-2 / 3}-\frac{8}{135} \kappa^{-14 / 3} \dot{\kappa}^{2}+\frac{2}{45} \kappa^{-11 / 3} \ddot{\kappa} .
$$

Therefore

$$
\zeta_{1}=2 \kappa^{-2 / 3}, \quad \zeta_{2}=\frac{1}{15} \kappa^{-2 / 3}-\frac{2}{135} \kappa^{-14 / 3} \dot{\kappa}^{2}+\frac{8}{45} \kappa^{-11 / 3} \ddot{\kappa} .
$$

Hence after integration by parts we have

$$
I_{1}=-2 \int_{0}^{L} \kappa^{2 / 3} d s, \quad I_{2}=\frac{1}{1080} \int_{0}^{L}\left(9 \kappa^{4 / 3}+8 \kappa^{-8 / 3} \dot{\kappa}^{2}\right) d s .
$$


For the purposes of $\S \S 8$ and 9 it is convenient to rewrite these formulas in terms of the angular coordinate $r$ :

$$
I_{1}=-2 \int_{0}^{2 \pi} \kappa^{-1 / 3} d r, I_{2}=\frac{1}{1080} \int_{0}^{2 \pi}\left(9 \kappa^{1 / 3}+8 \kappa^{-5 / 3}\left(\kappa^{\prime}\right)^{2}\right) d r
$$

where $\kappa^{\prime}=d \kappa / d r$.

Next we consider the general form of the higher invariants $I_{k}$. First observe that the space $\mathcal{T} \subset C^{\infty}(\mathbf{R} / 2 \pi \mathbf{Z})$ of all functions $\chi$, satisfying (2.8), (2.9) and therefore corresponding to convex regions, has a natural homotheity. Thus the transformation

$$
M_{R}: \chi(r) \mapsto R \chi(r)
$$

corresponds to the usual radial expansion on $\mathbf{R}^{2}$.

(4.9) Proposition. Under the transformation (4.8), the $I_{k}$ are homogeneous,

$$
I_{k}(R \chi)=R^{1-2 k / 3} I_{k}(\chi) \text {. }
$$

Each $I_{k}$ is the integral of a polynomial in the derivatives of $\chi$ up to order $k-1$, with coefficients constant multiples of powers (in general negative) of $\chi^{1 / 3}$.

Proof. We need to analyse the behaviour of $I(t)$, and to do this of $\zeta$ under the transformation (4.8). The basic observation is that the underlying homotheity

$$
\Omega \ni x \mapsto R x \in \Omega_{R}
$$

which corresponds to (4.8) is linear. If $(x, \sigma) \in B^{*} \partial \Omega$, then $(R x, \sigma) \in B^{*} \partial \Omega_{R}$ and

$$
\delta_{+, R}(R x, \sigma)=\left(R y, \sigma^{\prime}\right),
$$

if $\delta_{+}(x, \sigma)=\left(y, r^{\prime}\right)$. That is, the two boundary maps are intertwined by the conformal symplectic transformation, given in terms of the normalization (2.1) and corresponding arclength by

$$
T_{R}:(s, \sigma) \mapsto(R s, \sigma), T_{R}^{*} \omega_{R}=R \omega .
$$

If $\zeta \in C^{\infty}\left(B^{*} \partial \Omega\right)$ and $\zeta=T_{R}^{*} \zeta^{\prime}, \zeta^{\prime} \in C^{\infty}\left(B^{*} \partial \Omega_{R}\right)$, then the two Hamilton vector fields are related by.

$$
\left(T_{R}\right)_{*} H_{\zeta}=R H_{\zeta^{\prime}}
$$

The formula $\delta_{+}=\exp \left(-\zeta^{1 / 2} H_{\zeta}\right)$ transforms to $\delta_{+, R}=\exp \left(-R\left(\zeta^{\prime}\right)^{1 / 2} H_{\zeta^{\prime}}\right)$, so

$$
T_{R}^{*} \zeta_{R}=R^{2 / 3} \zeta \text {. }
$$

In the transformed region therefore, $T_{R}^{*} d z_{R}=R^{1 / 3} d z$,

$$
I_{R}\left(R^{2 / 3} t\right)=R^{1 / 3} I(t) \text {. }
$$

Thus $I_{k+1}\left(\Omega_{R}\right)=R^{1 / 3-2 k / 3} I_{k+1}(\Omega)$, which is just (4.11). The remainder of the proposition is easily established by a formal power series computation. 


\section{Length spectrum}

Reflected geodesics in the manifold with boundary $\Omega$ consist of (continuous) curves in $\bar{\Omega}$ which are the unions of straight line segments $\left[x, x^{\prime}\right], x, x^{\prime} \in \partial \Omega$ with consecutive segments satisfying the usual form of Snell's law:

$$
\begin{gathered}
x^{\prime}-x=D \theta,|\theta|=1, \quad x^{\prime \prime}-x^{\prime}=D^{\prime} \theta^{\prime},\left|\theta^{\prime}\right|=1, \\
\theta-\theta^{\prime}=c n\left(x^{\prime}\right), \quad c>0,
\end{gathered}
$$

where $n\left(x^{\prime}\right)$ is the inward normal to $\partial \Omega$ at $x^{\prime}$. Directly from this definition, the end points of the line segments of such geodesics form a sequence with

$$
(x, \theta),\left(x^{\prime}, \theta^{\prime}\right)=\beta(x, \theta), \cdots .
$$

Indeed this sequence determines the geodesic as a curve parametrized by arclength, up to a translation. Since the sequence (5.3) consists of outward pointing elements it is fixed by its projection into $B^{*} \partial \Omega$, where it is of the form

$$
p, \delta_{+} p, \delta_{+}^{2} p, \cdots \text {. }
$$

Thus a maximally extended geodesic of $\Omega$ is determined uniquely by an orbit of $\delta_{+}$in $B^{*} \partial \Omega$. The closed geodesics, corresponding to finite $\delta_{+}$-orbits, are of particular interest. Associated with each closed geodesic $g$, is an integer $n=n(g)$ given by the number of reflections. If $g$ is not a simple closed geodesic then $n=k \bar{n}$, where $k$ is the multiplicity, and $\bar{n}$ is the number of reflections along the simple geodesic. There is a second integer determined by $\mathfrak{g}$, its rotation number. Consistently choosing either the positive (counterclockwise) or negative orientation of $\partial \Omega$, two points $p, p^{\prime} \in \partial \Omega$ determine intervals $\left[p, p^{\prime}\right]_{ \pm}$in $\partial \Omega$ with $\partial \Omega=\left[p, p^{\prime}\right]_{+} \cup\left[p, p^{\prime}\right]_{-}$. In this way, $g$ determines two curves parametrized by arclength:

$$
c_{+}(s, \mathfrak{g})=\left[p, \delta_{+} p\right]_{+} \cup\left[\delta_{+} p, \delta_{+}^{2} p\right]_{+} \cup \cdots,
$$

and similarly for $c_{-}$. If $m_{ \pm}$is the number of times $c_{ \pm}$covers $\partial \Omega$, then $m_{+}+m_{-}=n$. The rotation number $m$ of $g$ is then

$$
m=\min \left(m_{+}, m_{-}\right) \leqslant n / 2 \text {. }
$$

(5.7) Proposition (Poincaré). Let $\Omega \subset \mathbf{R}^{2}$ be a strictly convex compact $C^{\infty}$ region. For each pair of positive integers $n, m \in \mathbf{N}$ with $2 m \leqslant n$, the set $\Gamma(m, n)$ of closed geodesics making $n$ reflections and with rotation number $m$ is nonempty.

Proof. (Outline only, see Birkhoff [2]). The definition of $m$ extends directly to any ordered subset of cardinality $n$ :

$$
\mathfrak{h}=\left\{p_{1}, p_{2}, \cdots, p_{n}\right\} \subset \partial \Omega .
$$

Defining the length by

$$
L(\mathfrak{h})=\sum_{i=1}^{n} \operatorname{dist}\left(p_{i}, p_{i+1}\right),
$$


where $p_{n+1}=p_{1}$, one can follow Poincare in showing that amongst all $\mathfrak{h}$ with $m$ and $n$ fixed the supremum of $L(\mathfrak{h})$ is attained, and this maximum occurs at a closed geodesic.

The convexity of $\Omega$ immediately shows that if $\mathfrak{g} \in \Gamma(m, n)$, then

$$
L(g)<m L_{\infty}=L_{\infty, m},
$$

where $L_{\infty}$ is the length of the boundary. More significantly, if $\mathfrak{g}_{m, n} \in \Gamma(m, n)$ with $m$ fixed, then

$$
\lim _{n \rightarrow \infty} L\left(g_{m, n}\right)=m L_{\infty} .
$$

In fact the curves $\mathfrak{g}_{m, n}$ approach the gliding geodesics consisting of $m$ circuits of $\partial \Omega$, provided only that the initial points of the curves are constrained to converge.

Now the length spectrum of $\Omega$ consists of the closure

$$
\mathcal{L}(\Omega)=\operatorname{cl}\left\{L \in R^{+} ; L=L(\mathrm{~g}), \mathrm{g} \text { a closed geodesic }\right\} \text {. }
$$

From (5.10) and Proposition 5.7, the $m L_{\infty}$ are contained in this closure. It follows readily that if the gliding geodesics are included, then the set $\{L(\mathfrak{g})\}$ is itself closed.

(5.11) Proposition [8]. If $B^{*} \partial \Omega$ is considered as an abstract manifold, with $\boldsymbol{\delta}_{+}$ and the symplectic structure given, then $\mathcal{L}\left(\delta_{+}\right)$can be recovered, i.e., $\mathcal{L}(\Omega)=$ $\mathfrak{L}\left(\boldsymbol{\delta}_{+}\right)$is a symplectic invariant.

Proof. (Outline only, see [8]). Choose a $C^{\infty} 1$-form $\alpha$ on $B^{*} \partial \Omega$ such that

$$
d \alpha=\omega, \delta_{+}^{*} \alpha \text { is continuous, } \int_{S_{+}^{*} \partial \Omega} \alpha=\int_{S_{-}^{*} \partial \Omega} \alpha .
$$

Such a form exists since $\alpha=\sigma d s$, defined in the canonical coordinates $(s, \sigma)$ dual to arclength, has these properties. For $\alpha=\sigma d s$, it follows by direct computation that if $g$ is a periodic orbit of $\delta_{+}$, then

$$
\delta_{+}^{*} \alpha-\alpha=d f_{\alpha}, \quad L(g)=\sum_{p \in \mathfrak{g}} f_{\alpha}(p) .
$$

In fact (5.13) is valid for any $C^{\infty} 1$-form $\alpha^{\prime}$ satisfying (5.12), since $\alpha^{\prime}-\alpha$ is exact, $\alpha^{\prime}-\alpha=d g$, and then

$$
\delta_{+}^{*} \alpha^{\prime}-\alpha^{\prime}=d f_{\alpha^{\prime}}, \quad f_{\alpha^{\prime}}=f_{\alpha}+\delta_{+}^{*} g-g .
$$

The sum in (5.13) is therefore independent of the choice of $\alpha$, proving the Proposition.

That $\mathcal{L}(\Omega)$ is a symplectic invariant of $\delta_{+}$leads one to expect a connection with the invariants $I_{k}$ discussed in $\S 4$ above. Not only does $\mathcal{L}(\Omega)$ determine the $I_{k}$ 's, but it suffices to know the asymptotic behaviour as $n \rightarrow \infty$ of a sequence 
$L\left(g_{m, n}\right)$ to be able to find the $I_{k}$. If $\Omega$ is a strictly convex planar region set

$$
t_{m, n}=\inf \{L(\mathfrak{g}) ; \mathfrak{g} \in \Gamma(m, n)\}, \quad T_{m, n}=\sup \{L(\mathfrak{g}) ; \mathfrak{g} \in \Gamma(m, n)\},
$$
and more particularly, $t_{n}=t_{1, n}, T_{n}=T_{1, n}$.

(5.15) Theorem. For each fixed $m \in \mathbf{N}$ and all $k$,

$$
\lim _{n \rightarrow \infty} n^{k}\left(T_{m, n}-t_{m, n}\right)=0 .
$$

There are constants $c_{k}$ such that if $\mathrm{g}_{1, n} \in \Gamma(1, n)$, then

$$
L\left(g_{1, n}\right) \sim L_{\infty}+\sum_{k=1}^{\infty} c_{k} n^{-2 k} \text { as } n \rightarrow \infty,
$$

and for any $p=1,2, \cdots$ the $c_{k}$ for $k \leqslant p$ determine algebraically the $I_{k}$ for $k \leqslant p$, and conversely.

Proof. Notice that (5.16) implies that the coefficients $c_{k}$ in (5.17) are independent of the choice of sequence $g_{1, n}$. It is clear that as $n \rightarrow \infty$ the reflected geodesics approach $\partial \Omega$ uniformly. From (3.13) it follows that $\zeta=$ $O\left(n^{-2}\right)$ on $g_{1, n}$ and that $\zeta$ is constant on the orbit $\mathfrak{g}_{1, n}$ up to order $n^{-k}$ for any $k$. More particularly, if $d z$ is the dual 1-form to the interpolating Hamiltonian $\zeta$, then

$$
\alpha=\zeta d z+d s
$$

satisfies (5.13), provided $\zeta$ is extended to $B^{*} \partial \Omega$ to have $d \zeta \neq 0$ and $\zeta=2$ on $S_{-}^{*} \partial \Omega$. In view of (3.3)

$$
\delta_{+}^{*} \alpha-\alpha=d\left(-\zeta^{3 / 2}+h+\delta_{+}^{*} s-s\right),
$$

where $h$ vanishes to infinite order at $S_{+}^{*} \partial \Omega$. Thus

$$
L\left(g_{1, n}\right)=\sum_{\mathfrak{g}_{1, n}}\left(-\zeta^{3 / 2}+h+\delta_{+}^{*} s-s\right)=L_{\infty}-n \zeta^{3 / 2}
$$

$\bmod n^{-k}$ for any $k$. Computing $I(\zeta)$ from (4.1) for a value of $\zeta$ taken on $\mathfrak{g}_{1, n}$ gives

$$
I(\zeta)=\sum_{j=1}^{n} \int_{\left[p_{j}, p_{j+1}\right]} d z \equiv n \zeta^{1 / 2} \quad\left(\bmod n^{-k} \forall k\right) .
$$

This shows that $\zeta=\zeta\left(g_{1, n}\right)$ has an asymptotic expansion as $n \rightarrow \infty$, determined by and determining the $I_{k}$. Substituting this into (5.18) demonstrates both (5.16) and (5.17), completing the proof of the theorem.

Not only are the lengths of the geodesics $g_{1, n}$ asymptotically well-behaved, but as $n \rightarrow \infty$, the distribution of points in $g_{1, n}$ is well-behaved asymptotically with respect to any one point. We shall not use such a result below, so simply state without proof. 
(5.19) Theorem. Suppose $g_{1, n} \in \Gamma(1, n)$. Then there are functions $g_{k}\left(\kappa, \cdots, \kappa^{(2 k)}\right)$ such that if $\mathrm{g}_{1, n}=\left\{p_{1}, \cdots, p_{n}\right\}$ then

$$
\int_{p_{j}}^{p_{j+1}} d s \sim \frac{1}{n} L_{\infty}+\sum_{k=1}^{\infty} n^{-2 k} \int_{p_{j}}^{p_{j+1}} g_{k}\left(\kappa, \cdots, \kappa^{(2 k)}\right) d s,
$$

uniformly as $n \rightarrow \infty$.

\section{Poisson relation}

The Poisson relation for a compact Riemannian manifold with geodesically convex boundary, such as a strictly convex region in Euclidean space, was derived in [1]. Thus, if $\sigma_{D}(\lambda)$ is the spectral enumerating measure

$$
\sigma_{D}(\lambda)=\sum \delta(\lambda-\bar{\lambda})
$$

summed over those $\bar{\lambda}$ for which $\bar{\lambda}^{2}$ is an eigenvalue of (1.1), with multiplicity, then the Fourier transform satisfies

$$
\operatorname{sing} \cdot \operatorname{supp} \cdot\left(\hat{\sigma}_{D}\right) \subset-\mathscr{L}(\Omega) \cup\{0\} \cup \mathcal{L}(\Omega),
$$

where $\mathcal{L}(\Omega)$ is the length spectrum, described above in $\S 5$. Furthermore, as a consequence of the formula [6, (1.3)], if there is precisely one closed geodesic (up to parameter translation) of length $L$, and this is differentially isolated, then

$$
L \in \operatorname{sing} \cdot \operatorname{supp} \cdot\left(\hat{\sigma}_{D}\right)
$$

It is the purpose of this section to show that, even without such an assumption of nondegneracy on the closed geodesics, such a converse, (6.3) to (6.1), holds for a large class of convex planar regions subject to a noncoincidence condition.

(6.4) Theorem. If $\Omega \subset \mathbf{R}^{2}$ is $C^{\infty}$, bounded and strictly convex, $t_{n}$ and $T_{n}$ are defined by (5.16) with $m=1$, and there exists $N=N(\Omega)$ such that if $n>N$

$$
L\left(g_{m^{\prime}, n^{\prime}}\right) \neq t_{n}\left(\operatorname{resp} . T_{n}\right), \quad \forall g_{m^{\prime}, n^{\prime}} \in \Gamma\left(m^{\prime}, n^{\prime}\right), \quad m^{\prime} \neq 1,
$$

then $t_{n} \in \operatorname{sing} \cdot \operatorname{supp} \cdot\left(\hat{\sigma}_{D}\right)$ (resp. $\left.T_{n} \in \operatorname{sing} \cdot \operatorname{supp}\left(\hat{\sigma}_{D}\right)\right)$. The same is true for Neumann or Robin boundary conditions.

As a first step in the proof of this theorem we recall briefly from [6] the proof of (6.2), (6.3) and specialize to two dimensions. The basic identity underlying (6.2) is

$$
\hat{\sigma}_{D}=\pi_{*} \Delta^{*} E,
$$


where $E$ is the fundamental kernel of the Dirichlet problem for the wave equation

$$
\begin{aligned}
& \left(D_{t}^{2}-\Delta_{x}\right) E(t, x, y)=0, \quad(t, x, y) \in \mathbf{R} \times \Omega \times \Omega, \\
& E(t, x, y)=0, \quad x \in \partial \Omega, \\
& E(0, \cdot)=0, \quad D_{t} E(0, \cdot)=-i \delta(x-y) .
\end{aligned}
$$

$\Delta: \mathbf{R} \times \Omega \hookrightarrow \mathbf{R} \times \Omega \times \Omega$ is the lift of the embedding of $\Omega$ as the diagonal in $\Omega \times \Omega$, and $\pi: \mathbf{R} \times \Omega \rightarrow \mathbf{R}$ is projection onto the first factor. On a manifold without boundary, (6.6), for the corresponding fundamental kernel, is sanctioned by wavefront set considerations [5]. For a boundary problem such as considered here, a more elaborate treatment is needed (see [1]) to deal with the gliding rays. We do not need to reexamine such arguments since the desired results are related to the geometry and analysis of hyperbolic boundary points. We can summarize this as follows.

Suppose $\varepsilon>0$ is given. Then there is a distribution

$$
E_{\varepsilon}^{\prime}(t, x, y) \in \mathscr{Q}^{\prime}\left(\mathbf{R} \times \mathbf{R}^{2} \times \mathbf{R}^{2}\right),
$$

which has the following property. If $\varphi$ is a defining function for $\partial \Omega, \varphi>0$ in $\Omega$, then provided $\rho \in C_{c}^{\infty}(\Omega)$ is equal to one on a sufficiently large compact subset of $\Omega$,

$$
\hat{\sigma}_{D}-\pi_{*} \Delta^{*}\left(\rho E_{\varepsilon}^{\prime}\right)-\int_{0}^{\infty}\left(\pi_{\varphi}\right)_{*} \Delta^{*}\left((1-\rho) E_{\varepsilon}^{\prime}\right) d \varphi \in C^{\infty}\left(\left(\delta, L_{\infty}-\varepsilon\right)\right),
$$

for $\delta<L_{\infty}$ fixed. Here $\pi_{\varphi}: \Omega \rightarrow \mathbf{R}$ is the fibration near $\partial \Omega$ given by $\varphi$, i.e., with fibres on which $\varphi$ is constant. Again in (6.8) the term $\pi_{*} \Delta^{*}\left(\rho E_{\varepsilon}^{\prime}\right)$ is sanctioned by wavefront set considerations, as is the term $\left(\pi_{\varphi}\right)_{*} \Delta^{*}\left((1-\rho) E_{\varepsilon}^{\prime}\right)$, and this depends smoothly on $\varphi$, as a distribution on $(\delta, L-\varepsilon)$, where $\delta=\delta(\Omega)>0$.

The distribution $E_{\varepsilon}^{\prime}$ can be taken to be Lagrangian, and represents up to a $C^{\infty}$ error term the part of the fundamental kernel of the Dirichlet problem away from the gliding rays, not only the part away from the boundary. We recall from [6] the geometry associated to, and some of the symbolic properties of, $E_{\varepsilon}^{\prime}$. The wavefront set of $E_{\varepsilon}^{\prime}$ is contained in the union

$$
\Lambda=\bigcup_{j} \Lambda_{j},
$$

where $\Lambda_{j} \in T^{*}\left(\mathbf{R} \times \mathbf{R}^{2} \times \mathbf{R}^{2}\right) \backslash 0$ is the relation defined by $j$-fold reflection. Thus

$$
(t, x, \zeta, \xi ; y, \eta) \in \Lambda_{j},
$$

if the geodesic through $(y, \eta)$ meets $\partial \Omega$ transversally and after $j$-fold reflection from the inside of $\partial \Omega$ (and finally possible extension beyond $\partial \Omega$ ) passes through $(x, \xi) \in T^{*} \mathbf{R} \backslash 0$ with the total length $t, \tau= \pm|\xi|$. 


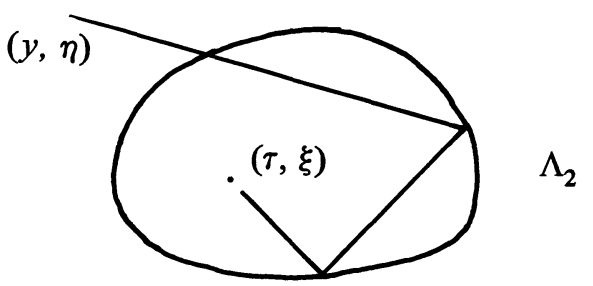

The construction in [6], following that of Chazarain in [3], shows that one can take

$$
E_{\varepsilon}^{\prime}=\sum_{\text {finite }} E_{j}, \quad E_{j} \in I^{-1 / 4}\left(\mathbf{R} \times \mathbf{R}^{2} \times \mathbf{R}^{2} ; \Lambda_{j}\right),
$$

where for each $\varepsilon$ only finitely many terms need to be taken, and the $E_{j}$ can be chosen independent of $\varepsilon$. The lower limit $\delta<L_{\infty}$ in (6.8) is just to ensure that $\Lambda_{j}$ satisfies a suitable transversality condition with respect to the $\pi_{\varphi}$. In fact, the $E_{j}$ can be taken to satisfy the wave equation in a neighborhood of $\partial \Omega$ :

$$
\left(D_{t}^{2}-\Delta_{x}\right) E_{j}(t, x, y)=\left(D_{t}^{2}-\Delta_{y}\right) E_{j}(t, x, y)=0, \varphi>-\delta,
$$

and to be fixed by suitable boundary conditions on $\partial \Omega$. Again from [6] we note that the densities $d t, d x, d y$ on $\mathbf{R}$ and $\Omega$ and a suitable parametrization of $\Lambda_{j}$ near $\partial \Omega$ can be used to trivialize the symbol (Maslov-Keller) bundle on $\Lambda_{j}$ so that the symbol of $E_{j}$ is $(-1)^{j} a_{j}, a_{j}>0$, with $a_{j}>0$ in a conic neighborhood of points of $\Lambda_{j}$ contributing to the singularities of $\hat{\sigma}_{D}$ within the interval $(\delta, L)$.

The main extension of [6] we use here is the following result which shows that $\hat{\sigma}_{j}$, the contribution of $E_{j}$ to $\hat{\sigma}_{D}$, is an oscillatory integral associated to the $j$ th iterate of the boundary map.

(6.11) Proposition. If $\Omega$ is a $C^{\infty}$ bounded and strictly convex planar region, there exists $N=N(\Omega)$ such that if $j>N$, then the contribution $\hat{\sigma}_{j}$ of $E_{j}$ to $\hat{\sigma}_{D}$ is of the form

$$
\hat{\sigma}_{j}(t)=\frac{1}{2 \pi} \int_{0} \int^{L_{\infty}} e^{i\left(t-\mu_{j}(s)\right) \tau} a_{j}(s, \tau) d s d \tau,
$$

where in terms of an arclength coordinate $s$ on $\partial \Omega$,

$$
\mu_{j}(s)=\left.\psi_{j}\left(s, s^{\prime}\right)\right|_{s=s},
$$

with $\psi_{j}\left(s, s^{\prime}\right)=L(\mathrm{~g})$, with $\mathrm{g}$ a j-fold reflected geodesic from $s$ to $s^{\prime}$, and $a_{j}$ is periodic in $s$, classical and elliptic of order zero, with principal part of the form $e^{i \pi r_{j} / 4} \alpha_{j}(s), \alpha_{j}(s)>0$.

Proof. We remark first that for $j$ large and $\left(s, s^{\prime}\right)$ a point near the diagonal in $\partial \Omega \times \partial \Omega$, there is a unique $j$-fold reflected geodesic making approximately one circuit of $\partial \Omega$ starting at $s$ and finishing at $s^{\prime}$. Thus $\psi_{j}$ in the statement 
above is well-defined for large $j,\left|s-s^{\prime}\right|<\varepsilon(j)$ and is a $C^{\infty}$ function. Moreover, it is clear from the results of [8] that $\psi_{j}$ is the generating function of the $j$ th iterate $\delta_{+}^{j}$ of the boundary map of $\partial \Omega$. Thus $(6.12)$ can be restated as

$$
\hat{\sigma}_{j}=\left(\pi^{\prime}\right)_{*}\left(\Delta^{\prime}\right)^{*} K_{j},
$$

where $K_{j} \in \mathscr{Q}^{\prime}(\mathbf{R} \times \partial \Omega \times \partial \Omega)$ is a Lagrangian distribution associated to the homogeneous lift of the boundary map:

$$
\begin{gathered}
K_{j} \in I^{0}\left(\mathbf{R} \times \partial \Omega \times \partial \Omega ; \Lambda_{j}^{\prime}\right), \\
\Lambda_{j}^{\prime}=\left\{\left(t, s^{\prime}, \tau, \sigma^{\prime}, s^{\prime \prime}, \sigma^{\prime \prime}\right) \in T^{*}(\mathbf{R} \times \partial \Omega \times \partial \Omega) \backslash 0 ; t=\psi_{j}\left(s^{\prime}, s^{\prime \prime}\right),\right. \\
\left.\delta_{+}^{j}\left(s^{\prime \prime}, \sigma^{\prime \prime} / \tau\right)=\left(s^{\prime}, \sigma^{\prime} / \tau\right),\left|s^{\prime}-s^{\prime \prime}\right|<\varepsilon(j), \tau \neq 0\right\} .
\end{gathered}
$$

The statements in the proposition about the symbol $a_{j}$ then follow from suitable statements about the symbols of the $K_{j}$ in (6.15).

Now (6.14) is a consequence of (6.8) with the additional use of (6.10). To exploit (6.10) first use the translation invariance in $t$ of the problem to note that

$$
\tilde{E}_{j}\left(t, x, t^{\prime}, y\right)=E_{j}\left(t-t^{\prime}, x, y\right)
$$

satisfies

$$
\left(D_{t}^{2}-\Delta_{x}\right) \tilde{E}_{j}=\left(D_{t^{\prime}}^{2}-\Delta_{y}\right) \tilde{E}_{j}=0 \quad \text { near } \Omega .
$$

For large $j$, the bicharacteristics making up $\Lambda_{j}$ are uniformly nearly, but never quite, tangent to $\partial \Omega$. Microlocally near any such curve $\beta$ here is a coordinate $r$ defined near $\pi(\beta) \in \mathbf{R}^{2}$ such that

(6.18) $r=0, \quad r=1 \quad$ on the two components of $\partial \Omega$ near $\pi(\beta)$, and such that $\left(D_{t}^{2}-\Delta_{x}\right)$ is noncharacteristic with respect to $r$. Then (6.17) can be microlocally factorized to

$$
\left(D_{r}-A(r)\right) E_{j} \equiv\left(D_{r^{\prime}}-A\left(r^{\prime}\right)\right) E_{j} \equiv 0,
$$

where $A(r) \in \psi^{1}\left(\mathbf{R}_{t} \times \mathbf{R}\right)$ is, microlocally near $\beta$, an elliptic pseudodifferential operator in the surface $r=$ constant, which is $t$-translation invariant. If we write $G\left(r, r^{\prime}\right)$ for the solution operator to the microlocal Cauchy problem for (6.19) (see for example [15])

$$
\left(D_{r}-A(r)\right) u \equiv 0,\left.\quad u\right|_{r=r^{\prime}}=u_{0} \Rightarrow u(r)=G\left(r, r^{\prime}\right) u_{0},
$$

then in terms of the coordinates $r$ and arclength on $\partial \Omega$,

$$
\tilde{E}_{j}\left(t, r, s ; t^{\prime}, r^{\prime}, s^{\prime}\right)=G(r, 0) G^{*}\left(r^{\prime}, 0\right) E_{j}^{\#},
$$


where $E_{j}^{\#} \in \mathscr{Q}^{\prime}(\mathbf{R} \times \partial \Omega \times \mathbf{R} \times \partial \Omega)$. Indeed, as is clear from the definition of $\boldsymbol{\delta}_{+}$,

$$
E_{j}^{\#} \in I^{\cdot}\left(\mathbf{R} \times \partial \Omega \times \partial \Omega ; \Lambda_{j}^{\prime \prime}\right),
$$

where $\Lambda_{j}^{\prime \prime}$ is obtained from $\Lambda_{j}^{\prime}$ in (6.16) by translation

$$
\begin{aligned}
& \Lambda_{j}^{\prime \prime}=\left\{\left(t, \bar{s}, \tau, \bar{\sigma}, t^{\prime}, s^{\prime}, \tau^{\prime}, \sigma^{\prime}\right) ;\right. \\
& \left.\quad 0=\tau+\tau,\left(t-t^{\prime}, \bar{s}, \tau, \bar{\sigma}, s^{\prime}, \sigma^{\prime}\right) \in \Lambda_{j}^{\prime}\right\} .
\end{aligned}
$$

Decomposing $\tilde{E}_{j}$ into suitable microlocal pieces and carrying out the restriction to $r=r^{\prime}$ in (6.8) and integration over $s=s^{\prime}$ give

$$
\begin{aligned}
\int \tilde{E}_{j}\left(t, r, s ; t^{\prime}, r, s\right) d s & =\int\left(G(r, 0) G^{*}(r, 0) E_{j}^{\#}\right)\left(t, s, t^{\prime}, s\right) d s \\
& =\int E_{j}^{\#}\left(t, s, t^{\prime}, s\right) d s,
\end{aligned}
$$

since $G^{*}(r, 0)=G(0, r)=G^{-1}(r, 0)$. Then we get (6.14) by taking

$$
K_{j}=E_{j}^{\#}\left(t, s ; 0, s^{\prime}\right)
$$

giving (6.15) as well. This proves the representation (6.12) since, as remarked above, $\left(t-\psi_{j}\left(s, s^{\prime}\right)\right) \tau$ is a nondegenerate phase function parametrizing $\Lambda_{j}^{\prime}$. The operators $G(r, 0)$ all have positive, classical symbols, and since $K_{j}$ is obtained by restriction from $E_{j}^{\#}$ the positivity results as stated for the symbol $a_{j}$ follow directly.

(6.26) Proposition. Any distribution of the form (6.12) with $a_{j}$ elliptic, classical and with positive principal symbol has

$$
\left\{\inf \left(\varphi_{j}\right), \sup \left(\varphi_{j}\right)\right\} \subset \operatorname{sing} \cdot \operatorname{supp} \cdot\left(\hat{\sigma}_{j}\right) \subset\left[\inf \left(\varphi_{j}\right), \sup \left(\varphi_{j}\right)\right],
$$

provided $j>N(\Omega)$.

Proof. The fact that $\operatorname{sing} \cdot \operatorname{supp} \cdot\left(\hat{\sigma}_{j}\right) \subset\left[\inf \left(\varphi_{j}\right), \sup \left(\varphi_{j}\right)\right]$ is of course standard, since the singular support of such an oscillatory integral is always contained in the set of singular values of the phase. The converse is an application (in the rather simple 1-dimensional case) of results of Soga [14] on singularities of degenerate oscillatory integrals. First if all the points at which $\sup \left(\varphi_{j}\right)$ is attained are nondegenerate maxima, then $\sup \left(\varphi_{j}\right) \in \operatorname{sing} \cdot \operatorname{supp} \cdot\left(\hat{\sigma}_{j}\right)$ follows from the lemma of stationary phase. Thus we can suppose that $\sup \left(\varphi_{j}\right)$ is attained at, at least one, degenerate maximum. Then Theorem 1 in Soga [14] shows that $\hat{\sigma}_{j}$ is still singular at $\sup \left(\varphi_{j}\right)$. A similar argument applies to the infimum of $\varphi_{j}$, completing the proof of the proposition.

Proof of Theorem (6.4). The hypothesis (6.5) is that $t_{n}$ is not the length of any closed geodesic with rotation number greater than one. If $n$ is large 
enough, it follows from (5.17) and $c_{1}<0$ that

$$
t_{n} \notin\left[t_{j}, T_{j}\right], j \neq n,
$$

and therefore that the only singular contribution to $\hat{\sigma}_{D}$ near $t_{n}$ comes from $\hat{\sigma}_{n}$. Applying Proposition (6.26) we deduce that $t_{n} \in \operatorname{sing} \cdot \operatorname{supp} \cdot\left(\hat{\sigma}_{D}\right)$. The same argument applies to show that $T_{n} \in \operatorname{sing} \cdot \operatorname{supp} \cdot\left(\hat{\sigma}_{D}\right)$ for large $n$ under the hypothesis (6.5) for $T_{n}$, completing the proof of the theorem.

\section{Spectral invariants}

Let $\sigma \subset C^{\infty}(\mathbf{R} / 2 \pi \mathbf{Z})$ be the set of all curvature functions $\chi$ satisfying (2.8), (2.9). Let $R \subset \mathcal{T}$ be the subset of curvature functions corresponding to regions with the following property:

(7.1) $L_{\infty}$ is not a limit point, from below, of $L(\Gamma(m, n)) \quad(m>1)$.

(7.2) Proposition. $\Re$ is dense in $\mathcal{\sigma}$ in the $C^{\infty}$ topology, and contains a $C^{1}$ neighborhood of the constants.

Proof. Notice that on any sequence of closed geodesics $g_{m, n}$ with $m \rightarrow \infty$, $L\left(\mathfrak{g}_{m, n}\right) \rightarrow \infty$, so that near any fixed element of $\widetilde{T}, L_{\infty}<L\left(\mathfrak{g}_{m, n}\right)$ if $m>M(\chi)$. Thus if $\Re_{1} \subset \mathcal{T}$ is fixed by the condition that

$$
L_{\infty} \neq L\left(g_{m, n}\right) \text { for any } m,
$$

then $\Re_{1} \subset \Re$. Moreover, the complement of $\Re_{1}$ characterized by the existence of a closed geodesic $\mathfrak{g}_{m, n}$ with $L\left(\mathfrak{g}_{m, n}\right)=L_{\infty}$ is clearly closed in the $C^{1}$ topology. Certainly the circles satisfy (7.3), so $\Re_{1}$, and hence $\Re$, contains a $C^{1}$ neighborhood of the constants.

To prove the first part of the proposition it is sufficient to note that the set $\Re_{2} \subset \mathcal{T}$ of curvature functions corresponding to domains for which all the $\Gamma(m, n)$ are finite and consist of only nondegenerate geodesics is itself open and dense in the $C^{\infty}$ topology, in $\mathcal{T}$. Thus $\mathscr{R} \supset \Re_{2}$ is dense. Of course, $\Re_{2}$ does not contain an open neighborhood of the circles.

(7.4) Theorem. For any convex region $\Omega$, with $\chi \in \Re$ the integral invariants $I_{k}(\Omega)$, are all uniquely determined by the spectrum of the Dirichlet problem. The same is true for Neumann or Robin boundary conditions.

Proof. The condition (7.1) defining $\Re$ requires that for some $\delta>0$,

$$
(L-\delta, L) \cap \mathcal{L}(\Omega) \subset \bigcup_{n} L(\Gamma(1, n)) .
$$

The combination of (6.2) and Theorem (6.4) shows that

$$
\bigcup_{n>N(\Omega)}\left\{t_{n}, T_{n}\right\} \subset \operatorname{sing} \cdot \operatorname{supp} \cdot\left(\hat{\sigma}_{D}\right) \cap(L-\delta, L) \subset \bigcup_{n}\left[t_{n}, T_{n}\right] .
$$


Moreover, from (5.16) the lengths of these intervals are rapidly decreasing as $n \rightarrow \infty$, whereas from (5.17) the distance between successive intervals is

$$
\frac{c}{n^{2}}+O\left(n^{-4}\right) \text { as } n \rightarrow \infty, c>0 .
$$

Thus the values of the $T_{n}$ for $n>N(\Omega)$ can be determined from $\operatorname{sing} \cdot \operatorname{supp} \cdot\left(\hat{\sigma}_{D}\right)$.

Of course this only determines the $T_{n}$ from an unknown initial point onwards, i.e., gives a sequence

$$
D_{k}=T_{k+p}, \quad k>\bar{k}
$$

However, it follows from (5.17) that $D_{k}$ has an asymptotic expansion as $k \rightarrow \infty$,

$$
D_{k} \sim L+\sum_{r=1}^{\infty} c_{r}(k+p)^{-2 r} \sim L+c_{1} k^{-2}-2 p c_{1} k^{-3}+O\left(k^{-4}\right)
$$

Since $c_{1}<0$ always, from (4.8), the value of $p$, and hence the values of all the invariants $c_{k}$, can be recovered from (7.6). The last part of Theorem (5.15) confirms that knowledge of the $c_{k}$ is equivalent to that of the $I_{k}$.

\section{Maximising $I_{1}$}

Up to a constant the first integral invariant is

$$
I_{2}^{\prime}=-\frac{1}{2} I_{1}=\int_{0}^{L} \kappa^{2 / 3} d s=\int_{0}^{2 \pi} \chi^{1 / 3} d r
$$

In view of Theorem (7.4), this is a spectral invariant of regions in $\Re$. Here we remark that amongst all strictly convex regions of fixed boundary length $L=L_{\infty}, I_{1}$ attains its maximum value only for the circles. Hence once more but in a different way one sees that the circular domains are spectrally determined (at least amongst the regions corresponding to $\Re$ ).

Indeed, by Hölder's inequality,

$$
-\frac{1}{2} I_{1} \leqslant\left(\int_{0}^{2 \pi} \chi d r\right)^{1 / 3}\left(\int_{0}^{2 \pi} d r\right)^{2 / 3}=L^{1 / 3}(2 \pi)^{2 / 3}
$$

with equality only for the constants, so the maxima is attained only for $\chi=L$, i.e., for the circle of circumference $L$. 


\section{Minimizing $I_{2}$}

The second invariant is

$$
\begin{aligned}
I_{2}^{\prime \prime} & =1080 I_{2}=\int_{0}^{L}\left(9 \kappa^{4 / 3}+8 \kappa^{-8 / 3} \dot{\kappa}^{2}\right) d s \\
& =\int_{0}^{2 \pi}\left(9 \chi^{-1 / 3}+8 \chi^{-7 / 3}\left(\chi^{\prime}\right)^{2}\right) d r
\end{aligned}
$$

where $\chi^{\prime}=d \chi / d r$. For fixed $L>0$ and $0<\varepsilon<(2 \pi)^{1 / 3} L^{1 / 6}$, set

$$
S_{\varepsilon}=\left\{\chi \in \mathcal{T} ; \int_{0}^{2 \pi} \chi d r=L, I_{1}^{\prime \prime}(\chi)=(2 \pi)^{2 / 3} L^{1 / 3}-\varepsilon^{2}\right\} .
$$

(9.3) Proposition. For each $L>0$ and $\varepsilon$ as above, the infimum of $I_{2}^{\prime \prime}(\chi)$ over $S_{\varepsilon}, \bar{I}_{2}^{\prime \prime}$, is attained. Moreover, for each $L>0$ there exists $\varepsilon_{0}>0$ such that for $0<\varepsilon<\varepsilon_{0}$ the minimum of $I_{2}^{\prime \prime}$ in $S_{\varepsilon}$ satisfies

$$
9(2 \pi)^{4 / 3} L^{-1 / 3} \leqslant \bar{I}_{2}^{\prime \prime} \leqslant 9(2 \pi)^{4 / 3} L^{-1 / 3}+\gamma \varepsilon^{2}+C \varepsilon^{4},
$$

where $\gamma=306\left(\frac{L}{2 \pi}\right)^{-2 / 3}$.

Proof. Certainly $S_{\varepsilon} \neq \varnothing$ for $0 \leqslant \varepsilon<(2 \pi)^{1 / 3} L^{1 / 6}$, although $S_{0}=\left\{\chi=\frac{L}{2 \pi}\right\}$. $I_{2}^{\prime \prime}(\chi)$ being positive is bounded from below on $S_{\varepsilon}$. Let $\chi_{n}, n=1, \cdots$, be a minimizing sequence. We shall show the existence of $\delta>0$ such that if $\kappa_{n}=1 / \chi_{n}$, then

$$
\kappa_{n} \in\left\{\kappa \in S_{\varepsilon} ; \kappa(r) \geqslant \delta, r \in \frac{\mathbf{R}}{2 \pi \mathbf{Z}}\right\} \quad n \gg 0 .
$$

This suffices to prove the existence of the minimum of $I_{2}^{\prime \prime}$ on $S_{\varepsilon}$, since the set in (9.5) is weakly closed in $H^{1}\left(\frac{\mathrm{R}}{2 \pi \mathrm{Z}}\right)$, on which $I_{2}^{\prime \prime}$ is an elliptic integrand.

Setting $\psi(r)=(\kappa(r))^{1 / 6}$, note that

$$
I_{2}^{\prime \prime}(\chi)=I_{2}^{\prime}(\psi)=9 \int_{0}^{2 \pi}\left(32\left(\psi^{\prime}\right)^{2}+\psi^{2}\right) d r .
$$

Thus if $\psi_{n}=\left(\kappa_{n}\right)^{1 / 6}$ is the minimizing sequence for $I_{2}^{\prime},\left\{\psi_{n}\right\}$ is bounded in the Sobolev space $H^{1}\left(\frac{\mathrm{R}}{2 \pi \mathrm{Z}}\right)$. Thus $I_{2}^{\prime}\left(\psi_{n}\right) \leqslant C^{2}$, set $\alpha_{n}=\inf _{r} \psi_{n}(r)$, attained at $r_{n}$. Then

$$
\left|\psi_{n}(r)-\psi_{n}\left(r_{n}\right)\right| \leqslant \int_{r_{n}}^{r}\left|\psi_{n}^{\prime}\right| d r \leqslant C\left|r-r_{n}\right|^{1 / 2}
$$

Thus

$$
L=\int_{0}^{2 \pi} \psi_{n}^{-6} d r \geqslant w\left(\sup _{\left|r-r_{n}\right| \leqslant w} \psi_{n}\right)^{-6} \geqslant w\left(\alpha_{n}+c w^{1 / 2}\right)^{-6}
$$

which shows that $1 / \alpha_{n}$ is bounded, proving (9.5). Hence the infimum is attained. 
To prove the bound (9.4) note that for small $\varepsilon$,

$$
\psi=\frac{L}{2 \pi}+\delta \cos (2 r)
$$

is in $S_{\varepsilon}$ for a choice of the constant $\delta$ of the form

$$
\delta=3 \sqrt{2} L^{5 / 6}(2 \pi)^{-4 / 3} \varepsilon+O\left(\varepsilon^{2}\right) .
$$

Then direct computation shows that

$$
I_{2}^{\prime \prime}(\kappa) \leqslant 9(2 \pi)^{4 / 3} L^{-1 / 3}+\gamma \varepsilon^{2}+O\left(\varepsilon^{4}\right),
$$

with $\gamma$ as stated in the proposition. This gives the upper bound in (9.4). The lower bound follows from Hölder's inequality. This completes the proof of the proposition.

Our goal is to show that any minimizer is a $\pi$-periodic function, rather than just a $2 \pi$-periodic function. We start with

(9.8) Lemma. If $\bar{\psi} \in S_{\varepsilon}$ is a minimizer for $I_{2}, 0<\varepsilon<\varepsilon_{0}$, then

$$
\int_{0}^{2 \pi} \bar{\psi} d t \geqslant(2 \pi)^{7 / 6} L^{-1 / 6}-\frac{7}{4}(2 \pi)^{1 / 2} L^{-1 / 2} \varepsilon^{2}+O\left(\varepsilon^{3}\right),
$$

where $\bar{\psi}=(\bar{\kappa})^{1 / 6}$.

Proof. The inequality

$$
\int_{0}^{2 \pi} \kappa^{-1 / 3} d r \leqslant\left(\int_{0}^{2 \pi}{ }^{-1} d r\right)^{3 / 7}\left(\int_{0}^{2 \pi}{ }^{1 / 6} d r\right)^{4 / 6}
$$

shows that, using the definition of $S_{\varepsilon}$,

$$
\begin{aligned}
\int_{0}^{2 \pi} \bar{\psi} d r & \geqslant\left(\int_{0}^{2 \pi} \bar{\kappa}^{-1 / 3} d r\right)^{7 / 4}\left(\int_{0}^{2 \pi} \bar{\kappa}^{-1} d r\right)^{-3 / 4} \\
& \geqslant(2 \pi)^{7 / 6} L^{-1 / 6}-\frac{7}{4}(2 \pi)^{1 / 2} L^{-1 / 2} \varepsilon^{2}+O\left(\varepsilon^{3}\right)
\end{aligned}
$$

as claimed.

(9.9) Corollary. If $\bar{\chi}$ is a minimizer for $I_{2}$ in $S_{\varepsilon}$, and $\varepsilon<\varepsilon_{0}, \bar{\psi}=(\bar{\chi})^{-1 / 6}$,

$$
I_{2}^{\prime}\left(\bar{\psi}-(2 \pi / L)^{1 / 6}\right) \leqslant C \varepsilon
$$

Proof.

$$
\begin{aligned}
I_{2}^{\prime}\left(\bar{\psi}-(2 \pi / L)^{1 / 6}\right)= & I_{2}^{\prime}(\bar{\psi})+9(2 \pi)^{4 / 3} L^{-1 / 3} \\
& -18(2 \pi / L)^{1 / 6} \int_{0}^{2 \pi} \bar{\psi} d r \\
\leqslant & \left(\gamma+\frac{63}{2}(2 \pi / L)^{2 / 3}\right) \varepsilon^{2}+O\left(\varepsilon^{3}\right) .
\end{aligned}
$$


This estimate shows that for any minimizer, $\bar{\chi}=1 / \bar{\kappa}$,

$$
\bar{\chi}=\frac{L}{2 \pi}+\nu
$$

where

$$
\|\nu\|_{H^{1}} \leqslant C \varepsilon
$$

Thus

$$
\begin{aligned}
I_{2}^{\prime \prime}(\bar{\chi})= & 8\left(\frac{L}{2 \pi}\right)^{-5 / 6} \int_{0}^{2 \pi}\left(\nu^{\prime}\right)^{2}+9(2 \pi)^{4 / 3} L^{-1 / 3} \\
& +2\left(\frac{L}{2 \pi}\right)^{-7 / 3} \int_{0}^{2 \pi} \nu^{2}+O\left(\varepsilon^{3}\right)
\end{aligned}
$$

since

$$
\int_{0}^{2 \pi} \bar{\kappa}^{-1 / 3}=\int_{0}^{2 \pi}\left(\frac{L}{2 \pi}+\nu\right)^{1 / 3}=(2 \pi)^{2 / 3} L^{1 / 3}-\frac{1}{9}\left(\frac{L}{2 \pi}\right)^{-5 / 6} \int_{0}^{2 \pi} \nu^{2}+O\left(\varepsilon^{3}\right),
$$

and similarly

$$
\int_{0}^{2 \pi} \nu^{2}=9\left(\frac{L}{2 \pi}\right)^{5 / 3} \varepsilon^{2}+O\left(\varepsilon^{3}\right)
$$

Therefore

$$
\begin{aligned}
I_{2}^{\prime \prime}(\bar{\chi})= & 9(2 \pi)^{4 / 3} L^{-1 / 3}+18\left(\frac{L}{2 \pi}\right)^{2 / 3} \varepsilon^{2} \\
& +8\left(\frac{L}{2 \pi}\right)^{-5 / 6} \int_{0}^{2 \pi}\left(\nu^{\prime}\right)^{2}+O\left(\varepsilon^{3}\right) .
\end{aligned}
$$

Now suppose the minimizer is given by $(9.10)$, then it follows that

$$
\int_{0}^{2 \pi}\left(\nu^{\prime}\right)^{2} d r \leqslant 36\left(\frac{L}{2 \pi}\right)^{5 / 3} \varepsilon^{2}+O\left(\varepsilon^{3}\right)
$$

Take a trigonometric expansion for $\nu$, normalized by translation to have no term $\sin (2 r)$,

$$
\nu=\delta \cos (2 r)+\sum_{k \geqslant 3}\left(s_{k}^{\prime} \cos (k r)+s_{k}^{\prime \prime} \sin (k r)\right),
$$

which shows in particular that

$$
\sum_{k \geqslant 3} k^{2}\left(s_{k}^{\prime}\right)^{2}+k^{2}\left(s_{k}^{\prime \prime}\right)^{2} \leqslant C \varepsilon^{2}
$$


(9.14) Lemma. For a minimizer of $I_{2}^{\prime \prime}$ on $S_{\varepsilon}$, put in the form (9.10), with $\nu=\delta \cos (2 r)+\nu_{e}+\nu_{0}$, where $\nu_{e}$ and $\nu_{0}$ are respectively the sums in (9.12) over $k \geqslant 4$ even and $k \geqslant 3$ odd,

$$
\left\|\nu_{e}\right\|_{H^{1}}, \quad\left\|\nu_{0}\right\|_{H^{1}} \leqslant C \varepsilon^{3 / 2}
$$

Proof. Suppose $\chi_{t}$ is a curve in $S_{\varepsilon}$ with $\chi_{0}=\bar{\chi}$, the minimizer, and tangent vector $\mu$ at $t=0$. Then

$$
0=\frac{d}{d t} I_{1}^{\prime}\left(\chi_{t}\right)_{t=0}=-\frac{1}{3} \int_{0}^{2 \pi} \bar{\chi}^{-4 / 3} \mu d r
$$

Since $\bar{\chi}^{-4 / 3}=\left(\frac{L}{2 \pi}\right)^{4 / 3}+\frac{4}{3}\left(\frac{L}{2 \pi}\right)^{1 / 3} \nu+O\left(\varepsilon^{2}\right)$, it follows from this that if

$$
\mu=\sum_{k \geqslant 4}^{\text {even }}\left(s_{k}^{\prime} \cos (k r)+s_{k}^{\prime \prime} \sin (k r)\right)+\tau \cos (2 r),
$$

then $\delta \tau=-\Sigma\left(s_{k}^{\prime}\right)^{2}+\left(s_{k}^{\prime \prime}\right)^{2}+O\left(\varepsilon^{3}\right)$, since $|\delta|>c \varepsilon, c>0$; this gives an admissible tangent vector.

Now, we also have

$$
0=\frac{d}{d t} I_{2}^{\prime \prime}\left(\chi_{t}\right)_{t=0}=\int_{0}^{2 \pi}\left(\frac{16}{9}\left(\frac{L}{2 \pi}\right)\right)^{-7 / 3} \nu^{\prime} \mu^{\prime}+\frac{2}{9}\left(\left(\frac{L}{2 \pi}\right)^{-7 / 3} \nu \mu\right)+O\left(\varepsilon^{3}\right),
$$

from which it follows that $\Sigma_{k \geqslant 4 \text {, even }}\left(k^{2}-4\right)\left(\left(s_{k}^{\prime}\right)^{2}+\left(s_{k}^{\prime \prime}\right)^{2}\right)=O\left(\varepsilon^{3}\right)$. That is, $\left\|\nu_{e}\right\|_{H^{1}} \leqslant C \varepsilon^{3 / 2}$. The odd part of the remainder can be handled similarly, proving the lemma.

Treating the error terms in such a computation a little more carefully we now proceed to

(9.18) Proposition. For $\varepsilon$ small, any minimizer of $I_{2}$ in $S_{\varepsilon}$ is $\pi$-periodic.

Proof. With the minimizer written in the form of Lemma (9.14), the objective is to show that $\nu_{0}=0$. Consider a variation of the type

$$
\chi_{t}=\frac{L}{2 \pi}+(\delta+\tau t) \cos (2 r)+\nu_{e}+(1-t) \nu_{0} .
$$

The necessary condition $\frac{d}{d t} I_{1}\left(\chi_{t}\right)=0$ at $t=0$ becomes $A \tau+B=0$, where

$$
\begin{aligned}
& A=\frac{1}{3} \int_{0}^{2 \pi} \cos (2 r)\left(\frac{L}{2 \pi}+\delta \cos (2 r)+\nu_{e}+\nu_{0}\right)^{-2 / 3}, \\
& B=-\frac{1}{3} \int_{0}^{2 \pi} \nu_{0}\left(\frac{L}{2 \pi}+\delta \cos (2 r)+\nu_{e}+\nu_{0}\right)^{-2 / 3} .
\end{aligned}
$$

Expanding in Taylor series and using the orthogonality of even and odd functions shows that

$$
A=-\frac{1}{9}(2 \pi) \delta\left(\frac{L}{2 \pi}\right)^{-5 / 3}(1+O(\varepsilon)), B=\frac{2}{9}\left(\frac{L}{2 \pi}\right)^{-5 / 3}\left(\int_{0}^{2 \pi}\left|\nu_{0}\right|^{2} d r\right)(1+((\varepsilon)) \text {. }
$$


Hence it is possible to obtain an admissible variation in $S_{\varepsilon}$ by taking

$$
\tau=\left(\int_{0}^{2 \pi}\left|\nu_{0}\right|^{2} d r\right)(1+O(\varepsilon)) /(\delta \pi)
$$

Computing directly, using the fact that $\int_{0}^{2 \pi}\left|\nu_{0}^{\prime}\right|^{2} \mathrm{dr} \geqslant 9 \int_{0}^{2 \pi}\left|\nu_{0}\right|^{2} d r$, one finds that with this variation,

$$
\frac{d}{d t} I_{2}\left(\chi_{t}\right) \leqslant-80\left(\frac{L}{2 \pi}\right)^{-7 / 3} \int_{0}^{2 \pi}\left|\nu_{0}\right|^{2} d r(1+O(\varepsilon)), \text { at } t=0
$$

which is only possible if $\nu_{0}=0$, proving the proposition.

(9.19) Proposition. For $\varepsilon>0$ small, the minimizer of $I_{2}$ in $S_{\varepsilon}$ is unique.

Proof. The arguments above apply to any minimizer. In particular, always for $\varepsilon<\varepsilon_{0}$ small enough, it follows from Lemma (9.14) that any minimizer can be normalized by translation to be of the form (9.10) with (9.15) valid. Then any two minimizers can be connected by a curve in $S_{\varepsilon}$ of the form (9.10) with uniform estimates (9.15). From the ellipticity of $I_{2}$ in $S_{\varepsilon}$ and the mountain pass lemma $I_{2}$ should then have another stationary point of the form (9.10), (9.15) and this cannot be a proper minimum. Computing the second variation of $I_{2}$, in directions transverse to the translation along the minimizer one easily obtains a contradiction since this partial Hessian is positive definite for small $\varepsilon$.

(9.20) Theorem. There exists $\varepsilon_{0}>0$ such that for each $0<\varepsilon<\varepsilon_{0}$, the infimum of $I_{2}$ on $S_{\varepsilon}$ defined by (9.2) is attained at a function $\chi \in \Re$, given by an elliptic integral, which corresponds to a convex region determined amongst regions with curvature in $\Re$ by its Dirichlet (or Neumann or Robin) spectrum.

Proof. Being $\pi$-periodic, the minimizer satisfies the Euler-Lagrange equation derived from the Lagrangian $I_{2}$ without the second two constraints in (2.10). That is,

$$
32 \dot{\kappa}^{2}=\kappa^{2}+a \kappa^{-2}+b \kappa^{-6}+c,
$$

for some constants $a, b, c$. Any solution of this is given by an elliptic integral. Since $L, I_{1}$ and $I_{2}$ are determined by the spectrum for regions in the noncoincidence set $\Re$ this completes the proof of the theorem.

\section{References}

[1] K. G. Anderrson \& R. B. Melrose, The propagation of singularities along gliding rays, Invent. Math. 41 (1977) 23-95.

[2] G. D. Birkhoff, Dynamical systems, Colloq. Publ., Vol. IX, Amer. Math. Soc., 1927.

[3] J. Chazarain, Formule de Poisson pour les variétés riemanniennes, Invent. Math. 24 (1974) 65-82.

[4] Construction de la parametrix du probleme mixte hyperbolique pour l'equation des ondes, C. R. Acad. Sci. Paris Ser. A.-B., 276 (1973) 1213-1215. 
[5] J. J. Duistermaat \& V. W. Guillemin, The spectrum of positive elliptic operators and periodic geodesics, Invent. Math. 29 (1975) 29-79.

[6] V. W. Guillemin \& R. B. Melrose, The Poisson summation formula for manifolds with boundary, Advances in Math. 32 (1979) 204-232.

[7] An inverse spectral result for elliptical regions in $R^{2}$, Advances in Math. 32 (1979) 128-148.

[8] $\_$A cohomological invariant of discrete dynamical systems, Christoffel Centennial Volume, Ed. P. L. Butzer and F. Feher, Birkhauser Verlag, Basel, 1981.

[9] M. Kac, Can one hear the shape of a drum? Amer. Math. Soc. Monthly, 73 (1966) 1-23.

[10] S. Marvizi \& R. B. Melrose, Some spectrally isolated convex planar regions, to appear.

[11] H. P. McKean \& I. M. Singer, Curvature and the eigenvalues of the Laplacian, J. Differential Geometry 1 (1967) 43-69.

[12] R. B. Melrose, Equivalence of glancing hypersurfaces, Invent. Math. 37 (1976) 165-191.

[13] R. Osserman, The isoperimetric inequality, Bull. Amer. Math. Soc. 84 (1978) 1182-1238.

[14] H. Soga, Oscillatory integrals with degenerate stationary points and their application to the scattering theory, Comm. Partial Differential Equations 6 (1981) 273-287.

[15] M. E. Taylor, Pseudodifferential operators, Princeton University Press, Princeton, 1981.

INSTITUTE FOR ADVANCED STUDY

MASSACHUSETTS INSTITUTE OF TeChNOLOGY 